\title{
CONTINUOUS PERSPECTIVE TRANSFORMATIONS AND THE PERCEPTION OF RIGID MOTION ${ }^{1}$
}

\author{
JAMES J. GIBSON AND ELEANOR J. GIBSON
}

\section{Cornell University}

An exploratory survey of the varieties of optical motion which could serve as stimuli for the perception of motions in the world $(6,7)$ suggested the hypothesis that one kind of geometrical motion in a plane yields an impression of a rigid motion in space but that any other kind of geometrical motion in a plane does not. The stimulus pattern was always a "texture," that is, a grouping of dark shapes on a light background. If, on a motion picture screen, it underwent a continuous sequence of perspective transformations in any of six ways, it gave a perception of a rigid surface moving in one of six ways - the three transpositions and three rotations which, in combination, exhaust the possibilities of mechanical movement. If it underwent continuous transformations not of this geometrical kind (but only a few examples were presented) it aroused perceptions of nonrigid or elastic surface motions, of the kind exemplified in the movements of organisms.

Of the six rigid phenomenal motions, three (rotation around the line of sight, transposition up or down, and transposition right or left) are

1 This experiment was reported by the first author as part of an address entitled Stimulation and Perception delivered as retiring President of the Division of Experimental Psychology, APA, in September, 1955. The work was supported in part by the Office of Naval Research under Contract NONR 401(14) with Cornell University. An early form of the apparatus to be described was constructed, and preliminary experiments were performed by $\mathrm{H}$. R. Cort. The writers are also obligated to Dr. O. W. Smith for ideas and assistance. induced by a stimulus which common sense would call motion; one (transposition along the line of sight) by a stimulus which common sense would call expansion or contraction; and only the other two (rotation around a horizontal or a vertical axis) by a stimulus which common sense would call a transformation. Optics, however, demands geometrical terms. All six projected motions are different parameters of continuous perspective transformation, and they are mathematically akin. Common sense tells us that the first three optical motions should give the perceptions they do (a motion yields a motion) and that the last three should not (how can a change of size or shape yield a motion?). The assumption is that a visual experience has to resemble visually the optical stimulus that produced it. But a better assumption is that experiences need only correlate with their stimuli, not replicate them, and the present hypothesis says that any continuous sequence of perspective transformations is the correlate of perceptually rigid motion.

There was in the film some evidence to suggest that this hypothesis must be qualified if the perspective transformations are those obtained with parallel projection instead of polar projection, that is, with the special case of transformations when the focus of projection is at infinity. The two apparent rotations around a horizontal or a vertical axis then seemed to become somewhat ambiguous as to rigidity or elasticity, and apparent reversals of direction 
of rotation appeared. The apparent approach or recession also fails of necessity in this case because the change of size of the stimulus disappears with parallel projection. If the above observations are verified, the hypothesis should specify perspective transformations with polar projection. Previous experimental work on the kinetic depth effect (14, 15) or on other appearances of depth in moving fields $(3,12)$ does not supply evidence for or against the amended hypothesis since in general the changes of shape studied in them were not polar projective. These experiments, moreover, are mainly concerned with what can be called the appearance of internal depth of an object, whereas what we are here talking about is the appearance of slant depth of the face of an object. The distinction is made clear in the film. The apparent motion in depth previously studied by Smith (13), however, is relevant to our hypothesis. One or two of Wallach's many experiments on the kinetic depth effect $(14$, p. $212 \mathrm{ff}$.) are relevant indirectly if the changes of such impoverished stimuli as line segments or angles are restated in terms of perspective transformations.

This hypothesis is comparable to, but different from, the principle involved in Wertheimer's "law of common fate" (16) in several respects. Both refer to some kind of motion in or of a grouping of spots or forms, but Wertheimer's law predicts the organization of a figure in the visual field, whereas this predicts the quality of rigidity of a surface or surface-like experience in space. Wertheimer's law seems to imply that the various parts of the complex are united by sharing a common motion (such as moving in the same direction with the same velocity) but this hypothesis asserts that any perspective transformation is a single motion mathematically, including the size and slant transformations where, analytically considered, every part moves in relation to every other. Wertheimer's law leads to experiments on "configurations of motions" $(9,12)$ in which each part of the complex undergoes components of translation or rotation but the part is not itself transformed; a geometrical transformation, however, is something that permeates every part as well as the whole of a texture, and the apparatus used in the present experiment satisfies this condition.

It might be noted that the problem of how we discriminate the rigidity of rotating solid objects and of approaching or receding solid objects in the environment is closely connected with the traditional problems of shape constancy and size constancy. Langdon has recently shown that the shape constancy of an object is considerably increased under conditions highly unfavorable for it when the object is made to rotate (10). Likewise the question of why we see a rigid environment when we move among the solid surfaces around us is closely connected with the traditional problem of space perception (8).

Aim of the experiment.-The experiment to be reported sought to answer four questions. First, does the appropriate parameter of continuous perspective transformations with polar projection always give the perception of the changing slant of a constant shape? Second, are the judgments of amount of change of slant away from the picture-plane in good psychophysical correspondence with the "extent" or "length" of the transformation sequence? Also, how variable are these judgments? Third, 
is the outcome dependent on or independent of the kind of shape or texture on which the transformation is imposed? Fourth, how accurate, if at all, is the judgment of slant away from the picture-plane when only the static end product of the transformation sequence is presented to $O$ but not the motion leading to it?

\section{Method}

\section{Apparatus and Stimuli}

The optical geometry of the apparatus used is shown in Fig. 1. The device can be termed a "shadow transformer." Essentially, it presents to an eye an optic array of limited scope within the boundaries of which either static patterns or continuous perspective transformations can occur. In this optic array, unlike those of everyday vision, the differential light intensities and their structure are under $E$ 's control; the pattern is the same for either eye, and the need for differential convergence and accommodation is eliminated. All the "cues for depth," in short, tend to determine a flat plane except those of form and motion, which are thus isolated for study. The source of this converging array is a window in a translucent screen.
This optical stimulus is artificially produced by the diverging ray sheaf from a point source of light, into which shadows are introduced by opacities of one sort or another attached to a transparent plane mount. Rotations or translations of the mount (on bearings or tracks outside the ray sheaf) yield corresponding transformation sequences of the shadow. This experiment utilized rotation on a vertical axis. The stimuli were the mirror reversals of these moving shadows, visible on the other side of the translucent screen. If an apparent rotation of a "virtual object" is induced by such a stimulus, it should always be opposite to the rotation of the shadow caster, without ambiguity.

The seated $O$, in a dimly illuminated room facing a large white surface, saw a luminous square window $36 \mathrm{~cm}$. on a side at a distance of $180 \mathrm{~cm}$., made of translucent plastic $\frac{1}{8}$ in. thick. The light source was fixed at the same distance behind the window as the eye was in front. It was a 300-w. Sylvania point source carbon arc lamp, but any lamp with a single filament of small diameter (up to $1 \mathrm{~mm}$. or more) will serve the purpose. The window was visibly flat. Binocular vision was permitted $O$ after preliminary work failed to show any difference between the use of one or two eyes. The mounts were transparent rectangular sheets of $\frac{1}{8}$-in. plastic, of such size $(30 \times 100 \mathrm{~cm}$.) that when they were centered and rotated on a turntable placed midway between the point

TRANSLUCENT SCREEN WITH SHADOW

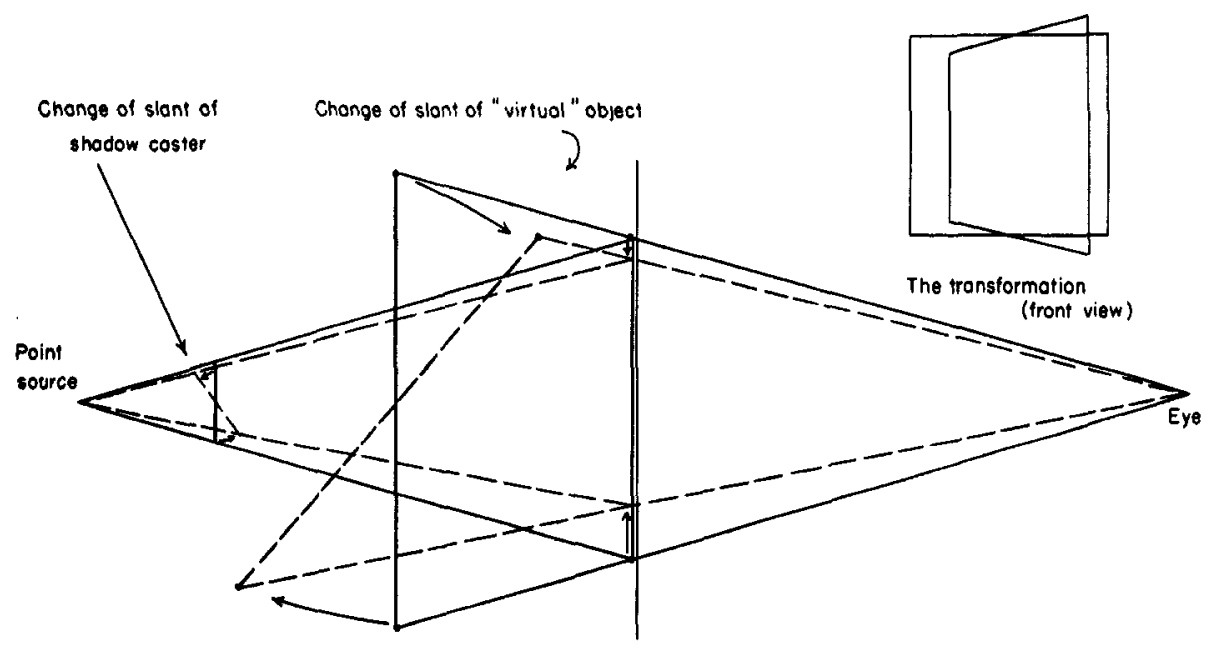

VIEW OF APPARATUS FROM ABOVE PRODUCING A SLANT TRANSFORMATION

FIG. 1. The shadow transformer. 




Fic. 2. Judgments of change of slant as a function of the length of the transformation sequence.

source and the window they could be turned $70^{\circ}$ from the parallel plane without the edges being projected within the window. The turntable could be rotated back and forth through an arc of variable length by an adjustable eccentric linkage, geared to a motor with a variable speed drive. A speed which gave 2-sec. cycles of semirotation was chosen, after exploration indicated that an optimum might be in this neighborhood, although the rate was not critical for the experiment. The quantitative variable of this experiment, then, was the "length" of the transformation sequence, as expressed in degrees of angular excursion of the turntable. We shall return to this point later. Five degrees of semirotation were presented: $15^{\circ}, 30^{\circ}, 45^{\circ}, 60^{\circ}$, and $70^{\circ}$. Each cycle began with and returned to the parallel plane.

The forms transformed. -The variety of forms, patterns, and textures that can be projected with this device has been suggested elsewhere (7). Four were used in the experiment: an amoeboid group of amoeboid dark shapes or spots (the irregular texture), a solid amoeboid contour form (the irregular form), a square group of dark squares (the regular texture), and a solid square (the regular form). Each was cut out of gummed paper and attached to the central area of a transparent mount so that its shadow was projected to the center of the square translucent window. With the mount parallel, the regular shadows extended $20 \mathrm{~cm}$. each way in the $36 \mathrm{~cm}$. square window, and the irregular shadows about the same. It may be noted that the "regular" stimuli are constituted of rectilinear contours and alignments and the "irregular" stimuli of randomly curved contours and alignments. There are also differences in symmetry, and perhaps other geometrical properties. The "forms" are bounded by a single closed contour and the "textures" by many closed contours; the total contour length is much greater in the latter stimuli. A texture might be described as a "form of forms," as distinguished from a form as such. These textures were, however, very "coarse"; there were 36 squares in the "platoon" and 36 "amoebas" in the "colony."

The variable protractor.-For recording judgments of change of slant, $O$ had before him a sort of protractor with its baseline parallel to the plane of the screen. It bore an adjustable pointer which could be moved to indicate an angle of semirotation. The top side was blank but the bottom side carried another pointer and a scale which could be read accurately by $E$ after each trial.

\section{Instructions and Procedure}

The experimental group.-Before receiving any formal instructions for the experiment, each $O$ was seated and told: "You see in front of you a sereen with a window in it which will be illuminated during the experiment. I will first show you a moving pattern of dark lines filling the window. If what you see is a movement of some kind of object, describe it."

A network (woven wire fencing of a common type) was then placed on the turntable and turned through various excursions. Although the question was intended to suggest neither a deformation in the plane nor a rigid rotation out of the plane, all $O$ s reported seeing the latter, and spontaneously reported different amounts of rotation. The suggestions in the following instructions were hence considered permissible:

"During the experiment proper, a dark form or pattern will appear in the middle of the window. It will seem to rotate back and forth on a vertical axis-to turn away from the plane of the screen and return. Your task is to judge how far it rotates, or the maximum angle it makes with the screen. Use the circular model in front of you to make this judgment."

One of the four patterns was then presented at one of the five degrees of transformation for $20 \mathrm{sec}$., which permitted $10 \mathrm{cycles}$ of stimulation. The $O$ had no difficulty in making his judgment during that interval. Twenty such trials (five for each pattern) in an order counterbalanced for the group were made, and then another 20 trials in reverse order to determine whether a practice effect would appear. The $O$ was not told his errors. There were $20 \mathrm{Os}$ in the group.

The control group.-A separate group of 30 $O_{8}$ was treated as similarly as possible except 
that the four stimulus patterns were motionless. Only the end product of each transformation sequence was presented, and only one degree of transformation was used-that with the mount at $60^{\circ}$. For the preliminary exposure, $O$ was shown a motionless pattern filling the window, half the group seeing the network of lines and the other half a less objective cloud-like pattern (this making no difference in the outcome) and he was asked if he saw an object of some sort. Then $O$ was told that he would see a form or pattern in the middle of the window. It might be parallel or slanted away from the screen. If he saw it slanted away from the plane he was asked to judge the angle it made using the model in front of him. Four trials were given (one for each pattern) in an order counterbalanced for the group.

\section{Results}

The experimental group.-The first question is whether all $O$ s saw the changing slant of a rigid shape. As stated above, all did at the outset. During the 40 trials which followed, each of considerable duration, many spontaneous descriptions were offered, and 8 of the 200 s observed at some stage that the display could be seen as a compression of a two-dimensional pattern. They were all psychologists. Twelve did not so report, and stated at the end that they had never observed it. The two-dimensional impressions did not persist long enough to prevent the requested judgments of changing slant. There was no difference in this respect between the regular or irregular forms or textures.

The second question is whether the judgments of change of slant are a function of the amount of change of form. The "length" of the transformation sequence is expressed as the inverse angular excursion of the shadow caster, and this variable is plotted on the horizontal axis of Fig. 2. The judgments are plotted on the vertical axis, each point representing a mean of 40 reproductions. The

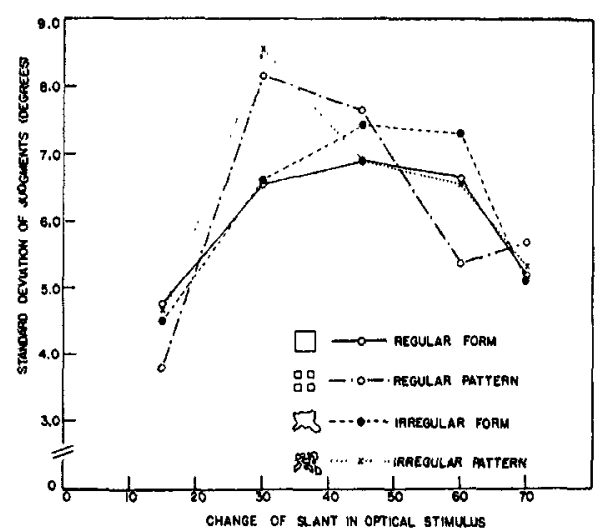

FIg. 3. Variability of judgments as a function of the length of the transformation sequence.

function is linear, except for a small tendency to underestimate (about 4 to $5^{\circ}$ ) the $15^{\circ}$ and $30^{\circ}$ angles. Even more striking, however, is the similarity of the functions for the regular and irregular and also for the single and multiple stimuli. This suggests that a transformation can be responded to as such, independently of what gets transformed.

A related question is how variable the judgments are within the group. Figure 3 shows the $S D$ 's of the judgments as a function of the change of slant "in" the stimulus. The variable errors range between about $4^{\circ}$ and $8^{\circ}$. They rise, but fall again as the maximum slant begins to approach $90^{\circ}$, at which limit a phenomenal surface becomes an edge.

The graph does not show any obvious differences between the errors obtained with the four kinds of pattern used. An analysis of variance (Table 1), however, is necessary to discover whether either multiplicity or regularity of the patterns is significantly related to errors of judgment. Multiplicity or "texturedness" is not. The effect of regularity 
TABLE 1

Analysis of Variance of Errors of Judgments of Change of Slant

\begin{tabular}{|c|c|c|c|}
\hline Source & $d f$ & MS & $F$ \\
\hline $\begin{array}{l}\text { Degree of excursion of } \\
\text { turntable }(A)\end{array}$ & 4 & 1437.07 & $13.12^{* *}$ \\
\hline Regular or irregular (R) & 1 & 320.68 & $5.56^{*}$ \\
\hline Form or texture $(\mathrm{T})$ & 1 & 72.90 & \\
\hline Subjects $(S)$ & 19 & 453.93 & \\
\hline Practice (P) & 1 & .53 & \\
\hline$A \times S$ & 76 & 109.47 & \\
\hline $\mathrm{R} \times S$ & 19 & 57.69 & \\
\hline$T \times S$ & 19 & 52.63 & \\
\hline$P \times S$ & $\begin{array}{r}19 \\
4\end{array}$ & $\begin{array}{l}23.07 \\
6054\end{array}$ & \\
\hline $\begin{array}{l}\mathrm{A} \times \mathrm{R} \\
\mathrm{A} \times \mathrm{T}\end{array}$ & $\begin{array}{l}4 \\
4\end{array}$ & $\begin{array}{l}60.54 \\
67.09\end{array}$ & $2.66^{*}$ \\
\hline $\mathrm{T} \times \mathrm{R}$ & 1 & 13.15 & \\
\hline$A \times S \times R$ & 76 & 12.69 & \\
\hline & & 25.24 & \\
\hline $\mathrm{T} \times \mathrm{R} \times S$ & 1 & 299.13 & \\
\hline
\end{tabular}

Note.-Only those interactions which appeared to be of some interest or were used for the $F$ tests (11, $p$, 330 and 338) are included in the Table.

$\begin{aligned} * \mathrm{P} & =.05 . \\ * * \mathrm{P} & =.01 .\end{aligned}$

is significant at only the $5 \%$ level. $^{2}$ The tendency is in the expected direction but is weak, considering predictions that might be made from Gestalt theory about "good form." It is strengthened, perhaps, by the significant interaction between angle and regularity which seems to reflect the tendency, barely noticeable in the graphs, for the irregular forms to depart slightly more from linearity at the larger angles. All the forms in this experiment were apparently good enough to carry the transformation, and it was this which mainly determined the judgments. This answers the third question. The form of the change seems to be what is important, not the form itself. A conception of the various forms that optical change may take is, however unfamiliar, probably necessary for an understanding of the perceptual process.

${ }^{2}$ The $5 \%$ level is probably not acceptable here, since some inhomogeneity of variance is evident in the data.
It may be noted from Table 1 that no significant practice effect appeared between the first and second blocks of 20 trials. They have been pooled in Fig. 2 and 3. The two halves of the data independently warrant the same conclusions, when the curve of Fig. 2 is plotted separately for them.

The control group.-The outcome of the control experiment was radically different inasmuch as the judgments of slant depended on the regularity of the form or texture presented. The irregular stimuli, in fact, generally appeared in the plane of the screen ( $85 \%$ of 60 judgments) while the regular stimuli generally appeared at a slant from the screen $(97 \%$ of 60 judgments). Even for the regular stimuli, however, the mean degree of slant perceived was only $24^{\circ}$ ( $S D$ about $12^{\circ}$ ) whereas for the moving regular stimuli the mean had been $61^{\circ}$ (SD about $\left.6^{\circ}\right)$. This is gross underestimation for the motionless and great accuracy for the moving stimuli. The underestimation of slant is consistent with previous research on static optical forms and optical textures under similar conditions. A trapezoidal form can sometimes arouse an impression of slant, but an exact linkage between the apparent shape and the apparent slant (a "psychological invariant") is not obtained (2). A static optical texture with a compression of texture on one meridian relative to the other induces a perception of surface slant, but even when the texture is regular the slant is underestimated, and when the texture is less regular the slant is more underestimated (4, p. 380).

The irregular form and the irregular texture displayed in this experiment were evidently not of such a kind as to appear slanted when altered by a slant transformation, since they generally still looked frontal. The $O$ s, 
TABLE 2

Means and $S D$ 's of Judgments of Change of Siant for Two O's

\begin{tabular}{|c|c|c|c|c|c|c|c|c|c|c|c|c|c|c|c|c|}
\hline \multirow{3}{*}{$\begin{array}{c}\text { Angular } \\
\text { Excursion of } \\
\text { Turntable }\end{array}$} & \multicolumn{4}{|c|}{ Solid Square } & \multicolumn{4}{|c|}{ Square of Squares } & \multicolumn{4}{|c|}{ Solid Amoeba } & \multicolumn{4}{|c|}{ Group of Amoebas } \\
\hline & \multicolumn{2}{|c|}{$o_{1}$} & \multicolumn{2}{|c|}{$\mathrm{O}_{2}$} & \multicolumn{2}{|c|}{$O_{1}$} & \multicolumn{2}{|c|}{$O_{2}$} & \multicolumn{2}{|c|}{$O_{1}$} & \multicolumn{2}{|c|}{$\mathrm{O}_{2}$} & \multicolumn{2}{|c|}{$o_{1}$} & \multicolumn{2}{|c|}{$O_{2}$} \\
\hline & $M$ & $S D$ & $M$ & $S D$ & $M$ & $S D$ & $M$ & $S D$ & $M$ & $S D$ & $M$ & $S D$ & $M$ & $S D$ & $M$ & $S D$ \\
\hline $\begin{array}{l}15^{\circ} \\
30^{\circ} \\
45^{\circ} \\
60^{\circ} \\
70^{\circ}\end{array}$ & $\begin{array}{r}8.4 \\
20.7 \\
47.3 \\
66.2 \\
69.2\end{array}$ & $\begin{array}{l}3.0 \\
4.9 \\
8.0 \\
5.4 \\
5.6\end{array}$ & $\begin{array}{r}9.4 \\
25.0 \\
43.2 \\
59.5 \\
60.8\end{array}$ & $\begin{array}{l}3.3 \\
7.1 \\
7.1 \\
6.1 \\
4.6\end{array}$ & $\begin{array}{r}9.1 \\
21.3 \\
39.8 \\
64.2 \\
72.2\end{array}$ & $\begin{array}{l}2.4 \\
6.2 \\
7.6 \\
4.8 \\
3.7\end{array}$ & $\begin{array}{r}7.9 \\
26.2 \\
44.1 \\
63.6 \\
67.5\end{array}$ & $\begin{array}{l}2.2 \\
4.4 \\
4.1 \\
4.9 \\
9.1\end{array}$ & $\begin{array}{r}9.7 \\
21.3 \\
45.5 \\
66.6 \\
76.6\end{array}$ & $\begin{array}{l}4.6 \\
4.8 \\
5.4 \\
5.0 \\
3.4\end{array}$ & $\begin{array}{r}9.2 \\
27.9 \\
44.4 \\
58.0 \\
65.2\end{array}$ & $\begin{array}{l}1.7 \\
9.0 \\
5.2 \\
5.6 \\
6.0\end{array}$ & $\begin{array}{r}8.7 \\
22.0 \\
41.8 \\
66.1 \\
78.2\end{array}$ & $\begin{array}{l}2.6 \\
4.6 \\
9.5 \\
7.7 \\
2.6\end{array}$ & $\begin{array}{r}9.3 \\
26.3 \\
43.2 \\
58.4 \\
68.2\end{array}$ & $\begin{array}{l}4.1 \\
6.2 \\
5.9 \\
5.2 \\
6.7\end{array}$ \\
\hline
\end{tabular}

Note.--Each cell in the table represents 10 judgments.

of course, had never seen them before transformation..$^{3}$ A truer statement of the matter is that the family of perspective transformations of the amoeboid stimuli has no unique member with immediately identifiable properties. The family of perspecțive transformations of the quadrilateral stimuli does have such a member-the square. At the outset there is one in this family which the other members can be transformations of, but none in the former family. Hence, rectilinear contour and alignment-a rectilinear structure-provide a primary basis for presumptions of slant which nonrectilinear structure does not.

One of the writers has argued that there exists a better basis than contour for presumptions or even perceptions of surface slant, namely, internal texture and its density variables (4). ${ }^{4}$

${ }^{3}$ An $O$ who had become familiar with the frontal aspect of the irregular form or texture (such as either $E$ ) could see anot'ier aspect as slanted. So also could an $O$ who had persistently observed these patterns undergoing continuous transformations. Presumably these $O$ 's had learned to identify the surface, i.e., to recognize a previously unfamiliar object in nonfrontal aspects. This, we believe, is Wallach's "memory effect" in the perception of tridimensional forms (15).

${ }^{4}$ In the study referred to, the correlate of slant was said loosely to be a "gradient" of texture density. The correlate might as well be
It might be expected, therefore, that the texture of amoeboid spots would induce slant more often than the amoeboid form, and the texture of squares more slant than the square. Neither expectation was fulfilled. The explanation may be that the textures used (36 elements, or only about 6 each way) were too coarse to make the density variables determinate.

Evidently a configuration with only the feeblest stimulating power for depth perception, or none at all, can nevertheless carry a transformation sequence which yields accurate depth perception. This answers the fourth question.

Individual results.-Time did not permit more than two judgments for each condition per $O$ in the experiment just reported. It was thought desirable to run a more exhaustive series in order to determine the extent of the variable error of the slant judgments for single individuals. Accordingly, two $O$ 's were run for five days each, so that 10 judgments per condition were available. ${ }^{5}$ Table 2

expressed as an unequal density along two meridians, or the ratio of these densities, or a special sort of compression of texture. These are all comprehended in the geometrical notion of a perspective transformation.

b Mr. John Hay kindly obtained these data. 
shows the means and $S D$ 's of these judgments. The means are strikingly like those for the group, especially for $O_{1}$, who underestimates the smaller angles and is more accurate or overestimates on the larger. The second $O$ tends to underestimate throughout. All the curves, when plotted, are close to linearity, and the particular pattern makes little difference. The $S D$ 's are of the same order as for the group.

\section{Discussion}

Kinetic depth effect and memory.Wallach believes that the kinetic depth effect of what we call a transformation sequence must be due to some effect of past experience on present momentary experience $(15$, p. 364). He argues that since any single member of the sequence looks flat in isolation, the present member has depth only because the memory traces of past members enter in to the present perception. He assumes that only the present member of the sequence can be a stimulus for the eye. This is a perfectly logical extension of the classical theory which strictly separates "traces" and "stimuli" as determiners of perception (or behavior). But does it not reduce the theory to an absurdity? Does a stimulus last for a second, a millisecond, or a microsecond? And what about the doctrine that "a stimulus is always a change"? Is it not theoretically preferable to suppose that a transformation is a stimulus in its own right, just as a nontransformation is a stimulus? Or, still better, that sequence, as well as pattern, is a variable of stimulation? Is it not better to take the risk that traces might vanish from psychological theory than the risk that the stimulus might vanish? Perhaps the addiction of Gestalt theorists to the concept of traces is what has prevented some of them from studying temporal forms as effectively as they have spatial forms.

Wallach has convincingly demonstrated (and the present observations confirm it) that a motionless nonsense pattern of a certain sort on a shadow screen which at the outset usually looks flat will usually look deep after $O$ has seen it in transformation. This result may well be considered a genuine effect of memory, or at least of recognition. But it is far from proving that memory traces are necessary for the perception of rigid motion. It might prove not an effect of memory on depth perception but an effect of memory on the depth interpretation of an ambiguous static picture. It is possible that the role of learning in space perception is quite a different affair, consisting not of the enriching of bare stimuli but the discriminating of rich stimuli.

The anchoring of a transformation sequence and the identifying of the object.A motionless pattern of sufficient irregularity appears in the plane of the screen even when its shadow-making pattern is slanted to the screen. What, then, would happen to the judgment of change of slant if the shadow caster were rotated between, say, $30^{\circ}$ and $60^{\circ}$ instead of between $0^{\circ}$ and $60^{\circ}$ ? Would $O$ then see the pattern as departing from and returning to the frontal parallel plane? Is the neat correspondence between length of transformation sequence and change of slant shown in Fig. 2 destroyed in these circumstances? These questions lead into fundamental problems of psychophysical scaling and shape constancy; complete answers cannot be given, but enough evidence has been obtained to be worth reporting here.

If the $30^{\circ}$ to $60^{\circ}$ cycle is presented to a naive $O$ with a rectilinear or regular pattern, judgments are made with some accuracy. The apparent surface does not begin and end in the parallel plane. If the cycle is presented with an irregular pattern, however, the naive $O$ reports a large change of slant which seems difficult to estimate, and the object does seem to return to the parallel plane. The crucial experience seems to be his first view of the motionless pattern. He sees an object but his first impression of it is an object thinner than the shadow-casting pattern. All observations so far suggest 
that if he is thus led to identify at the outset an object of the "wrong" shape, the judgments of change of slant will persistently be out of scale. His scale of slant will be stretched and displaced, as it were, until he is given the opportunity of anchoring it at $0^{\circ}$ and $90^{\circ}$ which, at the same time, enables him to identify the rigid shape of the object.

The size and distance of the virtual object are never determined by the optical stimulus in our experiment. Similarly, the slant and shape of the virtual object may be misdetermined at the outset by showing it, even briefly, as a continuous nontransformation-a static frontal pattern. This establishes a false shape constancy for the phenomenal object. When it rotates, anomalies of space perception will occur of the sort demonstrated strikingly by Ames with the "rotating trapezoidal window" (1). If care is taken by $E$, however, to avoid the procedure above, the evidence indicates that an intermediate transformation cycle can be correctly judged from the outset, whenever the length of the transformation sequence is sufficient. If a naive $O$ is first shown an anchored transformation sequence from $0^{\circ}$ to $90^{\circ}$, or even if he is first shown the motionless pattern at $45^{\circ}$ but is told not to assume that the object is necessarily in the plane of the picture, then a $30^{\circ}$ to $60^{\circ}$ cycle is judged approximately as such. The tentative conclusion is that a motion consisting of a perspective transformation sequence can determine both a definite rigid shape and a definite change of slant in perception, for a wholly unfamiliar object, without the need of any presumption whatever about the probable shape of the object based on memory (2).

It sounds very paradoxical to assert that a change of form of the stimulus can yield a constant form with change of slant in the percept. The paradox probably arises because two different meanings of the word "form" are employed (5), the first being geometrical and the second substantial. In any event, the assertion is inaccurate since the evidence now suggests that the form of the change of form of the stimulus is what determines the perception of rigid motion.

If the impression of surface rigidity in visual perception can be accounted for, the constancy of shape of objects is explained at the same time. The face of a unique solid object is ordinarily given not as a form but as a unique family of transformations to the eye. The difference between one solid object and another, in contrast, is not given as a relation of perspective transformation, nor is the difference between an earlier and a later state of a physically changing object. The perceptual problem of the recognizing or identifying of unchanging objects by their shape has to be approached in the light of these facts.

\section{SUMmary}

Continuous perspective transformations of varying length were presented in 2-sec. cycles to each $O$ on the visibly flat surface of a translucent screen. Judgments of the amount of change of slant of the apparently rigid object were in good correspondence with the length of the transformation sequence, without depending on the kind of pattern which carried the transformation. The patterns differed with respect to regularity vs. irregularity and form vs. texture. Regularity may have had a small effect on the variability of judgments but texturedness did not.

As a control, the same patterns were presented motionless at the end of a transformation sequence. In general the irregular patterns appeared to be in the frontal plane but altered in shape; the regular pattern appeared at some degree of slant, but the judgments were not accurate. Evidently impressions of changing slant are precise whereas corresponding impressions of unchanging slant are ambiguous or weak. Rectilinear contours and alignments seem to provide some basis for impressions of unchanging slant. A sequence of perspective transformations, on the other hand, seems to yield an impression of changing slant whether or not such regularity is present.

For irregular unfamiliar patterns there was evidence to suggest that the perceiving of the rigid shape of the virtual object is intimately connected with the perceiving of change of slant of the object. Misidentification of the shape at the outset was accompanied by anomalies in the perception of slant. 
The eye appears to be very sensitive to a continuous perspective transformation in the optic array. Psychophysical experiments are possible if the parameters of this stimulus are isolated and controlled.

\section{References}

1. Ames, A., JR. Visual perception and the rotating trapezoidal window. Psychol. Monogr., 1951, 65, No. 7 (Whole No. 324).

2. Beck, J., \& Grbson, J. J. The relation of apparent shape to apparent slant in the perception of objects. J. exp. Psychol., $1955,50,125-133$.

3. Fisichelli, V. R. Effect of rotational axis and dimensional variations on the rereversals of apparent movement in Lissajous figures. Amer. J. Psychol., 1946, $59,669-675$.

4. Grisson, J. J. The perception of visual surfaces. Amer. J. Psychol., 1950, 63, $367-384$.

5. Gisson, J. J. What is a form? Psychol. Rev., 1951, 58, 403-412.

6. Grison, J. J. Optical motions and transformations as stimuli for visual perception. (Motion picture film). State College, Pa.: Psychol. Cinema Register, 1955.

7. Grbson, J. J. Optical motions and transformations as stimuli for visual perception. Psychol. Rev., in press.
8. Gibson, J. J., Olum, P., \& Rosenblatt, F. Parallax and perspective during aircraft landings. Amer. J. Psychol., 1955, 68, 372-385.

9. Johannson, G. Configurations in event perception. Uppsala: Almqvist and Wiksell, 1950.

10. Langdon, J. The perception of a changing shape. Quart. J. exp. Psychol., 1951, 3, 157-165.

11. MoNemar, Q. Psychological statistics. (2nd ed.) New York: Wiley, 1955.

12. Metzger, W. Tiefenerscheinungen in optischen Bewegungsfeldern. Psychol. Forsch., 1935, 20, 195-260.

13. Smith, W. A. Sensitivity to apparent movement in depth as a function of "property of movement." $J$. exp. Psychol., 1951, 42, 143-152.

14. Wallach, H., \& O'Conneli, D. N. The kinetic depth effect. J. exp. Psychol., 1953, 45, 205-217.

15. Wallach, H., O'ConNell, D. N., \& Neisser, U. The memory effect of visual perception of three-dimensional form. J. exp. Psychol., 1953, 45, 360368.

10. Werthermer, M. Untersuchungen zur Lehre von der Gestalt. II. Psychol. Forsch., 1923, 4, 301-350.

(Received July 27,1956$)$ 